\title{
Fermentative Metal - Containing Bio-Nano complexes, Quantum - Chemical Models of Their Active Centers and Applications in Biotechnologies
}

\author{
Ludmila Uvarova ${ }^{1, *}$, Ekaterina Romanova ${ }^{2}$, Nikolai Korobov ${ }^{1}$, Elena Obukhova ${ }^{1}$ and Ekaterina Krasikova $^{1}$ \\ ${ }^{1}$ Moscow State Technological University "STANKIN", RU-127055, Moscow, Russia \\ ${ }^{2}$ FGBUN ICTS RAS, RU-127055 Moscow, Russia
}

\begin{abstract}
The general structure and the functional universal mechanism of the active centers of the fermentative metal-containing bio-nanocomplexes are discussed on the basis of the quantum-chemical approach using the conception "protein - machine". The process, proceeding taking place in the active centers structure is the result of their interaction with the substrates. The active centers quantum-chemical models represent the electron distribution among the complex molecular orbitals (MO). The interaction of the central ions with the ligands is carried out by the donor-acceptor mechanism: the ions provide the connection of the vacant orbitals and connection of the vacant orbitals and the ligands - the unshared electron pair. One of the coordination bonds is functional, using for the interaction with substrates. From the quantum-chemical point of view the functional mechanism of the bio-nanocomplexes is universal. In the final, it is reduced to the functional electron accepting and the leaving in the molecular antibonding orbitals of the complex, inducing the active center transitions from one state to another. The electron-conformational transitions, the active centers changes at its functioning, the role of its protein surrounding may be explained on a basis of the conception "protein - machine". The specific properties of the investigated bionanosystems are involved in the using of the internal valuable information, that is the process is a wave and occurs self-correlatively, and may be described by Kolmogorov-Petrovskii-Piskunov mathematical model. It is fair to say that the discussing bio-nanosystems are the natural intellectual tools, which may be used in the nanobiotechnologies and the spheres of its application are wide. It is necessary to conclude that the metalcontaining bio-nanocomplexes are the self-organizing, self-congruent, nonlinear, open systems.
\end{abstract}

\section{The analysis of the general idea about the metal-containing bionanocomplexes structure}

The fermentative metal-containing bio-nanocomplexes take part in the processes of the active life. Their molecular protein structures have been investigated for any length of time by using the physicochemical methods [1 - 9]. From these experiments some general conclusions can be drawn:

1). the fermentative molecules have the globular structures measured from ten to hundred nanometers.

2). the active centers built in the cooperative protein system - protein matrix.

$3)$. the active centers contain the central d-metal ions (for example, $\mathrm{Cu}(\mathrm{II}), \mathrm{Mn}(\mathrm{II}), \mathrm{Zn}(\mathrm{II}), \mathrm{Fe}(\mathrm{II}), \mathrm{Fe}(\mathrm{III})$ and so on).

4). the ferments active centers interact with the lowmolecular substances - the substrates (for example, O2, $\mathrm{H} 2 \mathrm{O} 2$ ).

5). the d-metal ions interact with the protein surrounding through the atoms $\mathrm{N}$ and $\mathrm{O}$ atoms of the amino acid residua, called ligands.

\section{The summarized results of the quantum-chemical modelling of the electron active centers structures}

The active centers carry the functional load. In spite of the diverse functions of the fermentative metalcontaining bio-nanocomplexes (catalytically functions, electrons and oxygen transport) the quantum-chemical approach has given the chance to determine and to make a model of the universal functioning mechanism, to construct the quantum models of the bio-nanocomplexes active centers $[8,9]$. The results received by the authors $[10-17]$ are summarized in the following paragraph.

The stereochemistry of the active centers is determined by the electronic configurations of central ions and the ligands nature. The active center complex can be attributed to the certain point symmetry group. The interaction of the central ion with the ligands is carried out by the donor-acceptor mechanism: the dmetal ion provides the connection of the vacant orbitals and the protein ligand - the unshared pairs of the electrons from the nitrogen or the oxygen atoms of the peptide groups. On the basis of the field ligands theory there can be made the model of the electron structure for 
any metal-containing active centers in the form of the complex molecular orbitals (MO) with the orbital electrons distribution over the orbitals. The universal mechanism of the active centers functioning - the electron arrival and electron leaving from the functional MO.

\section{The discussion of the fermentative bionanocomplexes structure scheme- model}

The active centers with the protein surrounding is a selforganizing, self-congruent, nonlinear, open system. Fig.1 shows the schematic representation of the bionanofermentative metal-containing complex structure model [19].

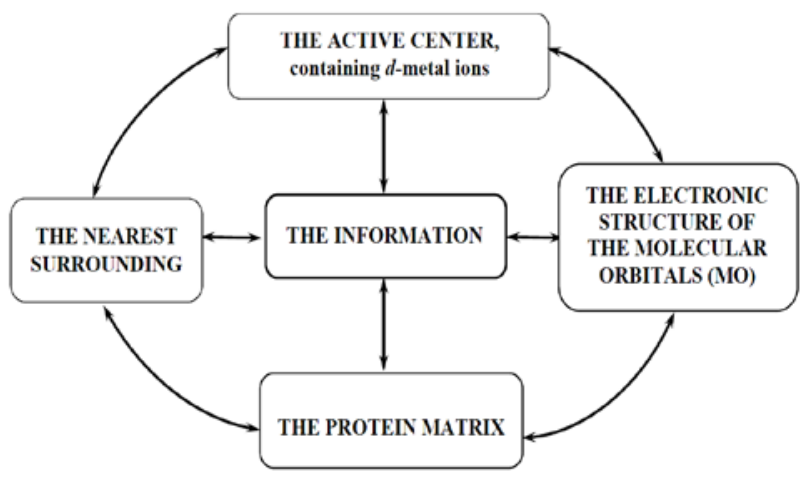

Fig.1. The scheme-model of the fermentative bionanocomplexes structure.

The process of the metal-containing active center functioning may be described in the following way. The interaction of the substrate with the active center produces its exciting state extending over the whole structure. This process is attended by the conformational changes which lie in the changes of the atoms space positions without the chemical bonds break. The conformational changes, as a result of the excitation propagation, have the cooperative character: that is, the displacement of separate atoms groups induce the displacement connected with them another groups connected with them.

This process may be considered as unidimensional analogy to "the work" of the mechanism-machine according to the "protein-machine" conception [10]. The conformational transitions induce the deformation of the system as a whole and its transition from the initial state to the new stable state. The system returns to the initial state after the end of interaction act. It is possible to have the transformation of the active center structure symmetry without destruction. The structure conservation is provided by the protein matrix.

The specific properties of the investigating bionanosystems are involved in the using of the internal valuable information and they are manifested on the quantum level.

\section{The mathematical model of the wave process in the metal-containing bionanocomplex active centers}

The process in the structure, shown in Fig.1, may be presented in the following way. The electron distribution among the complex molecular orbitals (MO), received on the basis of the quantum-chemical approach, determines the general active center conformational state, which may be described by the wave function. The information changes the system as a whole and induces the electron tunnel transport [10]. In this case the electron behaves as a wave. The system accepts information (a signal). There is a transformation to the excited state, spreading unidirectionaly, producing its conformational transition and the electron is leaving the system. Then the system returns to initial state. That process is a wave-like and occurs self-congruently and may be described by Kolmogorov-Petrovskii-Piskunov mathematical model [20].

The excited state propagation is described by the wave equation:

$$
\begin{gathered}
\frac{\partial u}{\partial t}=D \frac{\partial^{2} u}{\partial x^{2}}+f(u) \\
\text { where } f(u)=\beta u+\varepsilon u^{2}-u^{3}=0
\end{gathered}
$$

$f(u)$ - wave $D$ is the diffusion coefficient, $\beta>0(\beta$ and $\varepsilon$ are the parameters). The solutions of the equation (2) can be expressed by the following form:

$$
u_{1}=\frac{\varepsilon}{2}-\sqrt{\beta+\frac{\varepsilon^{2}}{4}}, u_{2}=0, u_{3}=\frac{\varepsilon}{2}+\sqrt{\beta+\frac{\varepsilon^{2}}{4}}
$$

The wave function can be represented as follows: $u(x, t)=u_{k}+w_{k}(x, t)(k=1,2,3)$. The perturbation $w$ may be considered to be little. It is possible to expand $f(u)$ in terms of $w: f(u)=f\left(u_{k}\right)+f^{\prime}\left(u_{k}\right) w_{k}$, as $f\left(u_{k}\right)=0$ for the wave function $w$ the wave equation can be written as follows:

$$
\frac{\partial w_{k}}{\partial t}=D \frac{\left(\partial^{2} w_{k}\right)}{\left(\partial x^{2}\right)}+\gamma_{k} w_{k}
$$

Where

$$
\begin{aligned}
& \gamma_{k}=f^{\prime}\left(u_{k}\right)= \begin{cases}-2 \sqrt{\frac{\varepsilon^{2}}{4}+\beta}\left(\sqrt{\frac{\varepsilon^{2}}{4}+\beta}-\frac{\varepsilon}{2}\right), & k=1 \\
\beta, & k=2 \\
-2 \sqrt{\frac{\varepsilon^{2}}{4}+\beta}\left(\sqrt{\frac{\varepsilon^{2}}{4}+\beta}+\frac{\varepsilon}{2}\right), & k=3\end{cases} \\
& \text { If } \beta>0, \text { so } \gamma_{1}<0, \gamma_{3}<0, \gamma_{2}>0 .
\end{aligned}
$$

The equation (4) is linear, its coefficients $D, \gamma_{k}$ are independent of the coordinate $x$ and the time. Its solution can be written as follows:

$$
w_{k}(x, t)=w_{k, 0} \exp (p t+i q x)
$$


Substituting the equation (6) into (4), it can be obtained for the parameters $p$ and $q$ the following relations:

$$
p=\gamma_{k}-D q^{2}, k=1,2,3,-\infty<q<+\infty
$$

According to (6) and (7) in case of $k=1, k=3 p<0$ for all $q$ meanings; for $k=2, u_{2}=0, p=\beta-D q^{2}$ and $p>$ 0 on some interval $q \in\left(-\sqrt{\frac{\beta}{D}} ;+\sqrt{\frac{\beta}{D}}\right)$. Therefore, small perturbation for the solution $u_{1}$ and $u_{3}$ are damping exponentially, and for $u_{2}=0-$ is growing. Consequently, the solutions $u_{1}$ and $u_{3}$ are stable, $u_{2}$ is unstable. The transition takes place between the states, which conforming to the solutions $u_{1}$ and $u_{3}$.

It should be noted that the fermentative metalcontaining bio-nanocomplexes are the natural intellectual devices, capable to react to the information (signals). Its retrieval and then the fulfillment of the specific action are the results of the functional parameters change.

In brief it may be said that the fermentative metalcontaining bio-nanocomplexes are the natural intellectual devices. Every such bio-nanocomplex has its "program" and substrate ("key to the lock"), functioning according to the conception "protein-machine" [10]. Discussing bio-nanosystems may be used in the nanobiotechnologies and the internal energy and information transportation. The producing of the intellectual materials on a basis of the unique natural biosystem is investigated presently.

On the basis of the calculating model it was demonstrated that the weak electromagnetic field interactions with the fermentative metalcontaining biocomplexes follow the pattern seen in the bionanocomplexes interactions with the substrates [11]. Thus it may be proposed that it is possible to exert control over the biocomplexes active centers functioning.

\section{The information properties of the bionanocomplexes}

The information problem is one of the actual problem at the present time. By the term problem it is meant the spectrum of questions, relating to the origin, the characteristics, the behavior of the information. There are very many definitions of the information notion and it implies, as a rule, that there is no-accurate view of the information as a phenomenon.

The last part of the 20th century was marked by the information explosion and the necessity to develop the scientific and may be universal theory of the information was created [21].

There has been discussed information method of approach in different spheres from biology and psychology to neurophysiology, linguistics, art criticism, in the literature.

Nowadays the informing properties of a biosystems are voted indisputably and are determined by their structures and functions which are interdependent. The biosystem specificity consists of a high degree of the coordination and coherence of the functioning processes



Fig.2. The scheme of the types of the information [20].

It is suggested that the specific properties are defined by intrinsic factor considered as the intrinsic information. The notion "information" is the subject of considerable literature. The determinations are numerous and varied. It has become clear during the past ten years, that the information is not a substance or energy. In the work [22] the answers to many questions, concerning the information are introduced. So, information is defined as a memorized choice of one option from some possible and equivalent ones. The researchers discuss problems such as obtaining and generating information, valuable information, the hierarchy of the information levels, the conditional, unconditional and memorized information, micro- and macroinformation. There were considered the different dynamic systems, including the information processes for biology in respect to the dynamic information theory. For the bionanocomplexes the valuable information holds much biological significance, which is dictated by the biocomplexes functions.

When the globular structure is being formed of the metalcontaining bionanocomplexes there is constructed the ordered structure - active centers, over which the valuable information is concentrating. It is necessary to have several stable stationary states for the information to be memorized. Having obtained information the active center is switching to the particular stable state. So the conformational state becomes monostable. The active center reverts to the original state upon the completion of the active center act. The information value is defined by the following expression [22]:

$$
V=\log _{2}\left(\frac{p^{\prime}}{p}\right)
$$

where $p^{\prime}$ - probability of the achievement of the purpose before obtaining the reception of the information, and $p$ - after [20]. The transition of the active center from one to the other state determines the informing process and connects with the intrinsic information.

In biosystems the concept of the valuable information is the basic concept. In fermentative systems the valuable information is concentrated in the field of the active center which represents «an information kernel», determining spatial structure and function 
bionanoclusters. Hence, complexity and specificity of enzyme is defined not only by structure, but also by the presence of the valuable information.

\section{Conclusion}

In the paper there have been discussed the biosystems d-metalcontaining ferments, having the globular structure, containing the active centers carrying the functional load. The active centers quantum-chemical models represent the electron distribution among the complex molecular orbitals (MO). The functioning of the active centers is reduced to the arrival of an electron and the exit of an electron from the system. The value of the information is of great importance for similar biosystems.

Their specific properties are defined by a high extent coordination, proceeding processes coherence. The functioning of active centers can be seen with the help of internal factors, so-called inside information. Also the metalcontaining bionanocomplexes are the selforganizing, nonlinear nanosystems.

It is concluded that the specific properties of the investigating bionanosystems are involved in the using of the internal valuable information, that the process is wave and occurs self-correlatively and may be described by Kolmogorov-Petrovskii-Piskunov mathematical model.

From the quantum point of view each information state of the active center, i.e. the electron distribution on the functional complex MO represent a single whole quantum state described by the general wave function $\Psi$. As a result of the information obtaining the system changes as a whole, that leads to electron removing which moves discretely (chaotically). Discrete movement of electron is a movement of a wave package which behaves as a wave therefore it breaks a potential barrier and leaves the system.

The evolution of the information notion, the most disseminating interpretation of the information notion, the functional role of the information are issues under study. These matters remain open, and the fact that it is impossible to construct the scientific picture of the world without solving these problems. It is possible to draw a conclusion that specificity of biologically-active metal containing nanocomplexes is determined by their informative properties which are shown at a quantum level.

The research was is supported by the Ministry of Science and Higher Education of Russia (Project №1.7706.2017/8.9). We thank Russian Science Foundation (grant №18-71-00137) and the Russian Ministry of Science and Education (grants 1.6198.2017/6.7 and 1.7706.2017/8.9) for support. This work was carried out using equipment provided by the Center of Collective Use of MSUT "STANKIN".

\section{References}

1. A. Kowalsky, J. Biol. Chem. 244, 6619 (1966)

2. J. Halpern, Pure Appl. Chem. 20, 59 (1969)
3. Y.P. Callman, Accounts Chem. Res. 1, 136 (1968)

4. L.E. Andreasson, T. Vänngard, Biochem. Biophys. Acta 200, 247 (1970)

5. P. Hambright, Coordination Chem. Rev. 6, 247 (1971)

6. W.H. Walker, P. Hemmerich, V. Masscy, Eur. J. Biochem. 13, 258 (1970)

7. Techniques and Topics in Bioinorganic chemistry. M.: Mir (1978)

8. J.B. Bersuker, Electronic structure and properties of coordination compounds. L.: Chemistry (1996)

9. Yu. Papulov, D. Papulova, The Molecule Structure and the physical properties. Tver: TSU, 168 (2010)

10. D.S. Chernavskii, N.M. Chernavskaya, «Protein machine». The biological macromolecular construction, (1999)

11. L.Yu. Vasil'eva, L.A. Uvarova, E.Yu. Romanova, The meso- and nanoobjects modeling in different mediums and fields, (2010)

12. L.Yu. Vasil'eva, Kluwer Academic, Plenum Publishers. 175, (2001)

13. L.Yu. Vasil'eva, E.Yu. Romanova, Articles. 11, 44 (2005)

14. L.Yu. Vasil'eva, E.Yu. Romanova, J. MSUA, 1, 34 (2005)

15. L.Yu. Vasil'eva, E.Yu. Romanova, Articles Math. Comp. Educ. 12, 197 (2014)

16. L.Yu. Vasil'eva, E.Yu. Romanova, Articles. 9, 40 (2006)

17. L.Yu. Vasil'eva, E.Yu. Romanova, J. NNSU, series Math. model. and optimal management. 13 (2006)

18. L.Yu. Vasil'eva, E.Yu. Romanova, Nova Science Publishers Jnl. 427 (2014)

19. L.A. Uvarova, E.Yu. Romanova, Articles. 19, 124 (2018)

20. N.V. Karlov, N.A. Kirichenko, Oscillations, Waves. Structures. M.: Physmatlit 242 (2003)

21. L.Yu. Vasil'eva, L.A. Uvarova, E.Yu. Romanova, Articles spc Academic North Charleston, USA, 3, 131 (2014)

22. D.S. Chernavskii, M. Book House Librokom, 15 (2009) 